\title{
A2 IDENTIFICATION OF A NEW CITRULLINATED EPITOPE ON FILAGGRIN FOR THE EARLY DIAGNOSIS OF RHEUMATOID ARTHRITIS
}

E Szarka, ${ }^{1}$ F Babos, ${ }^{2}$ A Magyar, ${ }^{2}$ F Hudecz, ${ }^{2}$ G Nagy, ${ }^{3}$ G Sármay ${ }^{1,4}{ }^{1}$ Department of Immunology, Eötvös Loránd University, Budapest, Hungary; ${ }^{2}$ HAS-ELTE Research Group of Peptide Chemistry, Budapest, Hungary; ${ }^{3}$ Buda Hospital of Hospitaller Brothers of St. John, Budapest, Hungary; ${ }^{4}$ Immunology Research Group of the Hungarian Academy of Sciences at ELTE, Budapest, Hungary

10.1136/ard.2010.129577b

Background Anti-citrullinated protein antibodies (ACPA) are sensitive and specific markers for diagnosis and prognosis in rheumatoid arthritis (RA). Citrullination is a post-translational modification of arginine by deimination, induced by peptidylarginine deiminase. It is a physiologically occurring phenomena during apoptosis, inflammation or keratinisation. Citrullination has been observed in different synovial proteins, including fibrinogen, vimentin and collagen. Antibodies specific for cyclic citrullinated filaggrin peptides (CCP) were detected in RA sera and anti-CCP positivity is widely used for diagnostic purposes. However, to determine the ACPAreacting epitopes on vimentin and new epitopes on filaggrin would be useful in the diagnosis of anti-CCP2 seronegative patients.

Our aim was to develop new tools for the detection of ACPA and thus for the early diagnosis of RA by the use of clearly defined epitopes on filaggrin, vimentin and collagen.

Methods First the authors used conventional solid-phase peptide synthesis (Fmoc strategy) carried out on 'MULTIPIN NCP' (Chiron Mimotopes Peptide System) non-cleavable kit. Citrullinated peptides and the unmodified counterparts containing arginine were synthesised on the pins in order to compare their respective reactivities. The authors have used these peptides-on-pins in an 'indirect' ELISA and ACPA was determined in the sera of RA and non-RA patients using anti-IgG plus IgM secondary antibodies. To validate the data obtained by multi-pin ELISA, the peptides were also synthesised in a Cor N-terminally biotinylated form by SPPS, according to Fmoc/ tBu strategy. The crude product was purified by reversed-phase chromatography. The structure of the peptide was proved by electron spray ionisation mass spectrometry and amino acid analysis.

Results Comparing short sequences of filaggrin, vimentin and collagen the authors have found that only two 5-mer and a 19-mer citrullinated filaggrin peptides were recognised by the RA sera, with a sensitivity and specificity that was comparable with the currently used tests. To validate our results, a 5-mer filaggrin peptides was re-synthesised either in a C-terminal or a N-terminal biotinylated form. These peptides were fixed to ELISA plates precoated with neutravidin. The RA sera samples specifically recognised the C-terminally biotinylated 5 -mer filaggrin peptide, while showed no reaction with the $\mathrm{N}$-terminally biotinylated ones. The authors could not find any correlation between the reactivity profile of the 5-mer peptide and the values of anti-CCP test, suggesting that the 5 -mer C-terminally biotinylated filaggrin peptide represents a new epitope recognised by ACPA.

Conclusion Identification of the new filaggrin epitope could be important for the development of novel diagnostic tools for RA. 